Supporting Information For:

\title{
Role of the Weak Interactions during the TNP Detecting Process of a Fluorescein-Based Sensor
}

Lei Liu ${ }^{\mathrm{ab}}$, Bingqing Sunc*, Ran Ding ${ }^{\mathrm{c}}$, Yueyuan $\mathrm{Mao}^{\mathrm{c}}$

a College of Chemical and Materials Engineering, Anhui Science and Technology University. Fengyang 233100, China

b State Key Laboratory of Molecular Reaction Dynamics, Dalian Institute of Chemical Physics, Chinese Academy of Sciences, Dalian, 116000, China

c College of Resource and Environment, Anhui Science and Technology University. Fengyang 233100, China

Corresponding Author: Bingqing Sun, Email: sunbq@ahstu.edu.cn

Contents

Figure S1, S2, S3

Figure S4, S5

Figure S6, S7

Figure S8, S9

Figure 10

Table S1, S2
Page Number

$\mathrm{S} 2$

S3

S4

S5

S6

S6 


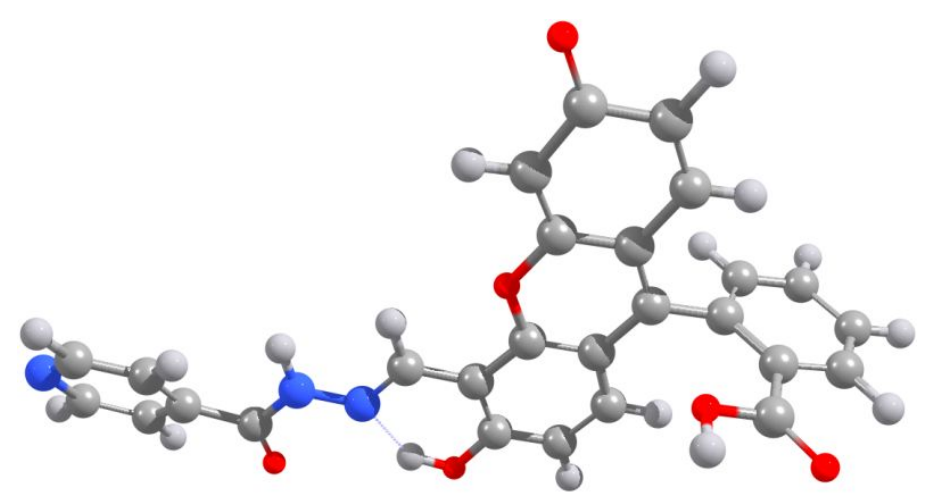

Figure S1. Molecular structure calculated by Fu et al.

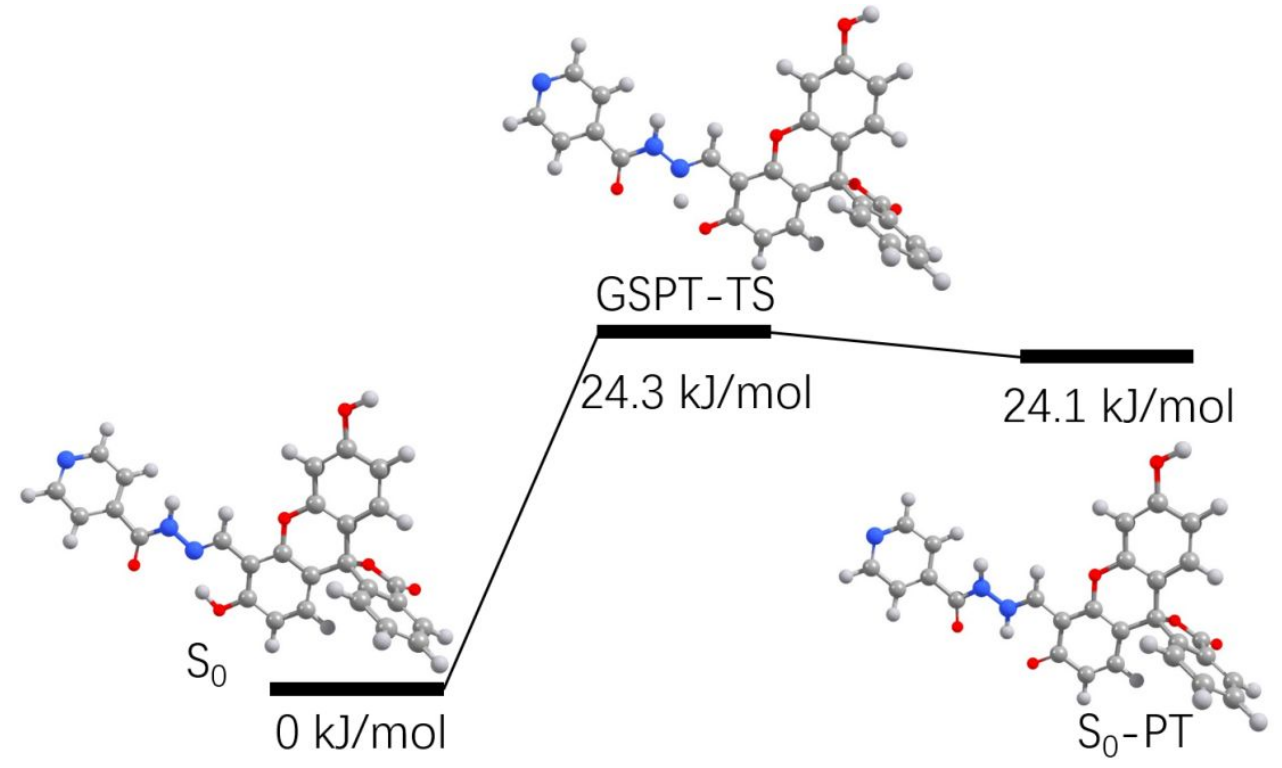

Figure S2. Energy diagram for the GSIPT process.

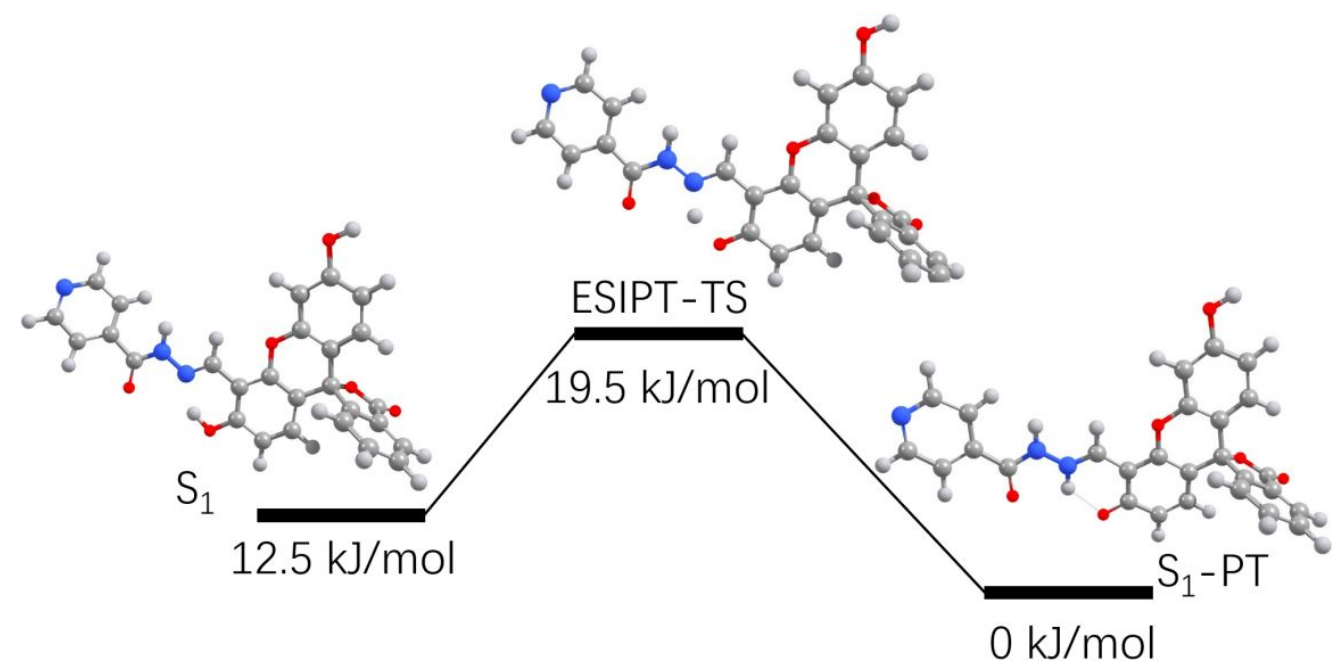

Figure S3. Energy diagram for the ESIPT process. 


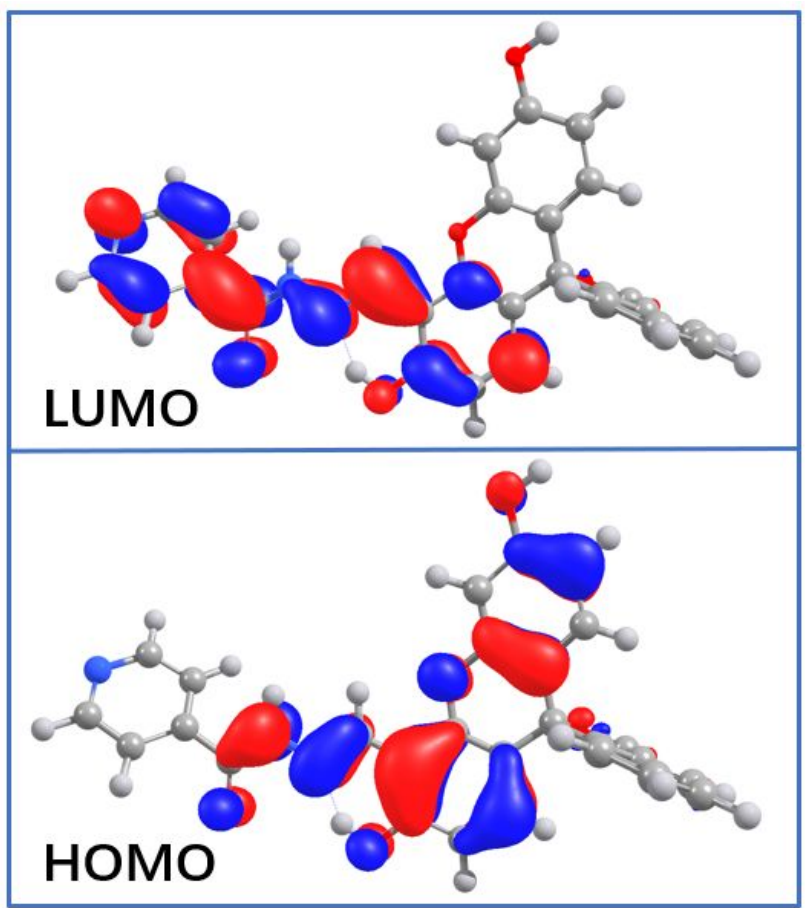

Figure S4. Molecular orbitals related to the excitation process of the sensor (obtained at CAM-B3LYP/TZVP theory level).

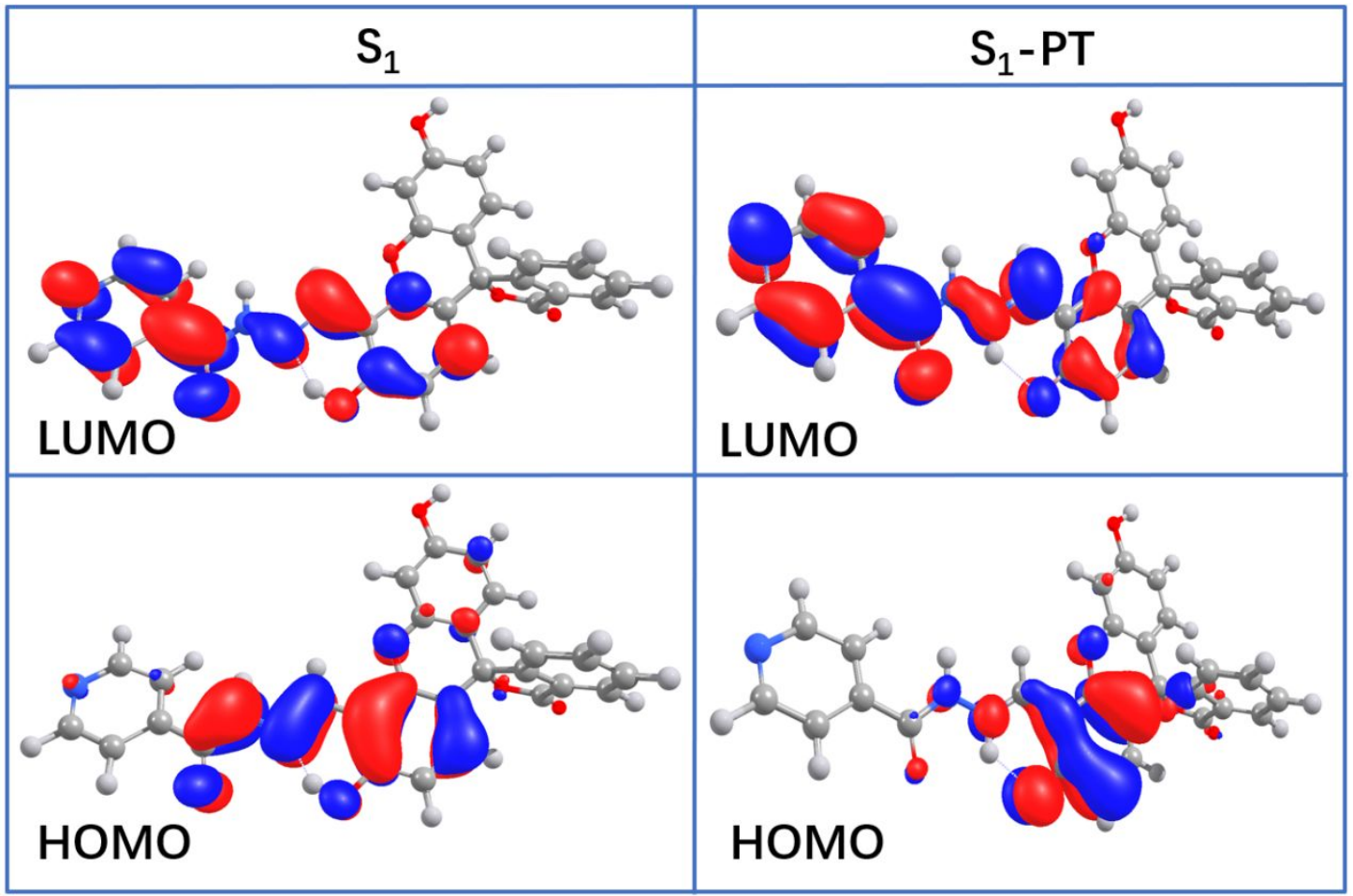

Figure S5. Molecular orbitals related to the emission process of the sensor (obtained at CAM-B3LYP/TZVP theory level). 


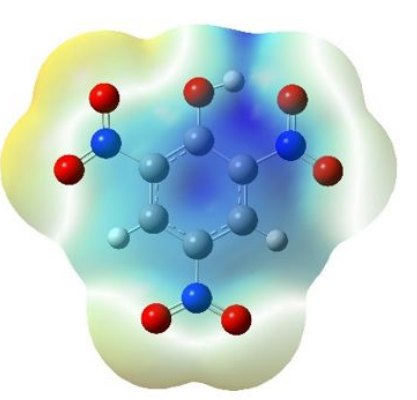

(a)

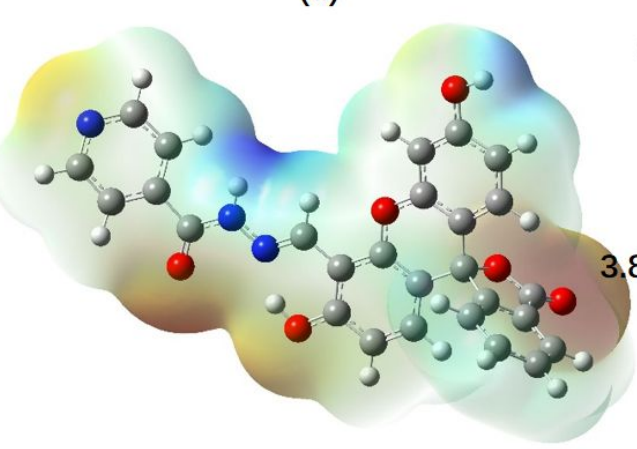

(b)
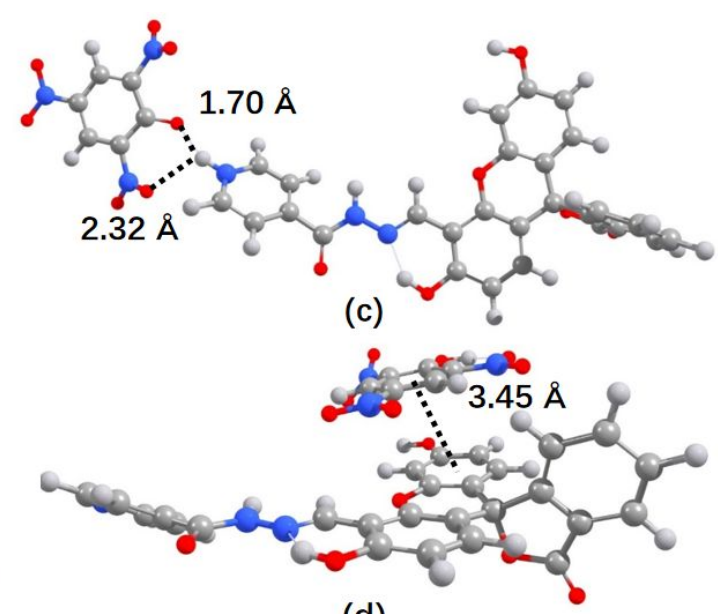

(d)

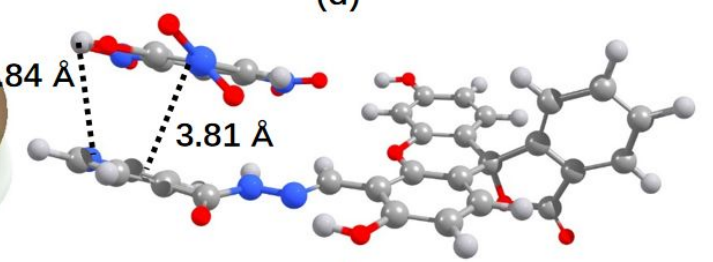

(e)

Figure S6. Electron static potential (ESP) surfaces of the analyte (a) and sensor (b); geometries of the $\mathrm{hb}-\mathrm{S}_{0}$ binding model (c) and the $\mathrm{pp}-\mathrm{S}_{0}$ binding model (d).
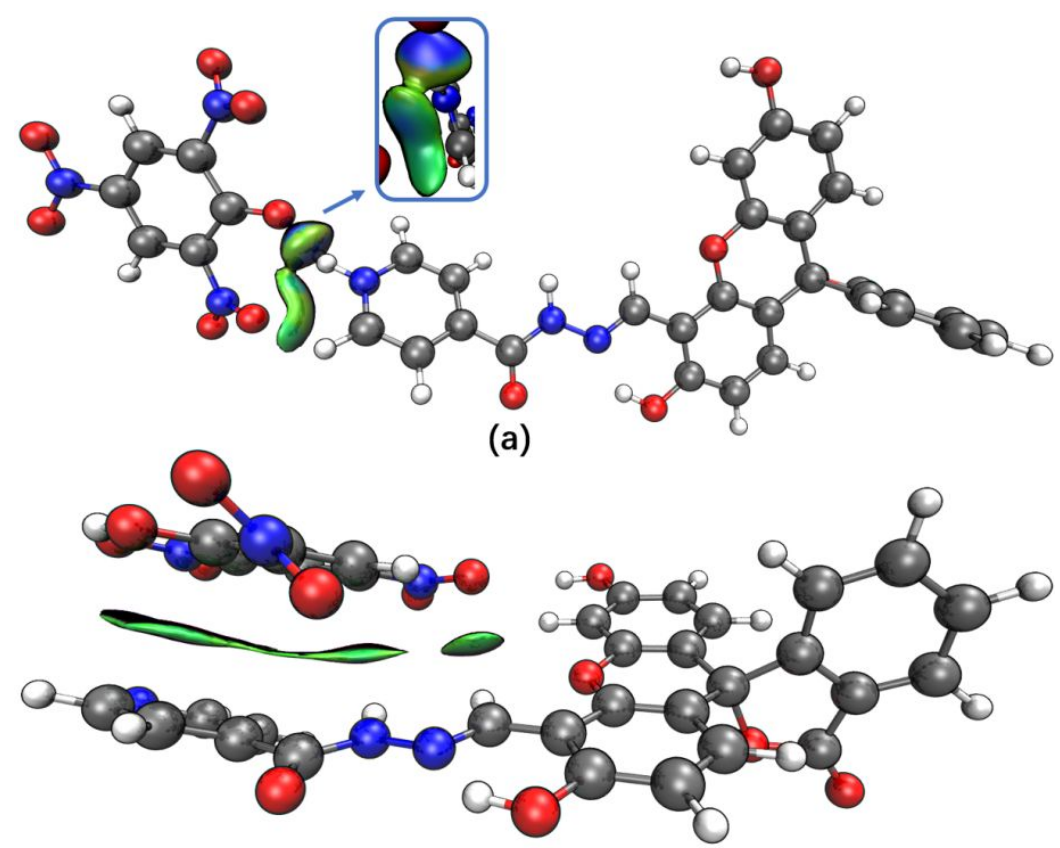

(b)

Figure $\mathrm{S} 7$. (a) IGM iso-surfaces for $\mathrm{hb}-\mathrm{S}_{0}$ binding model; (b) IGM iso-surfaces for $\mathrm{pp}-\mathrm{S}_{0}$ binding model (green region represents Van der Waals force, blue region represents strong attraction, e.g., hydrogen bond). 

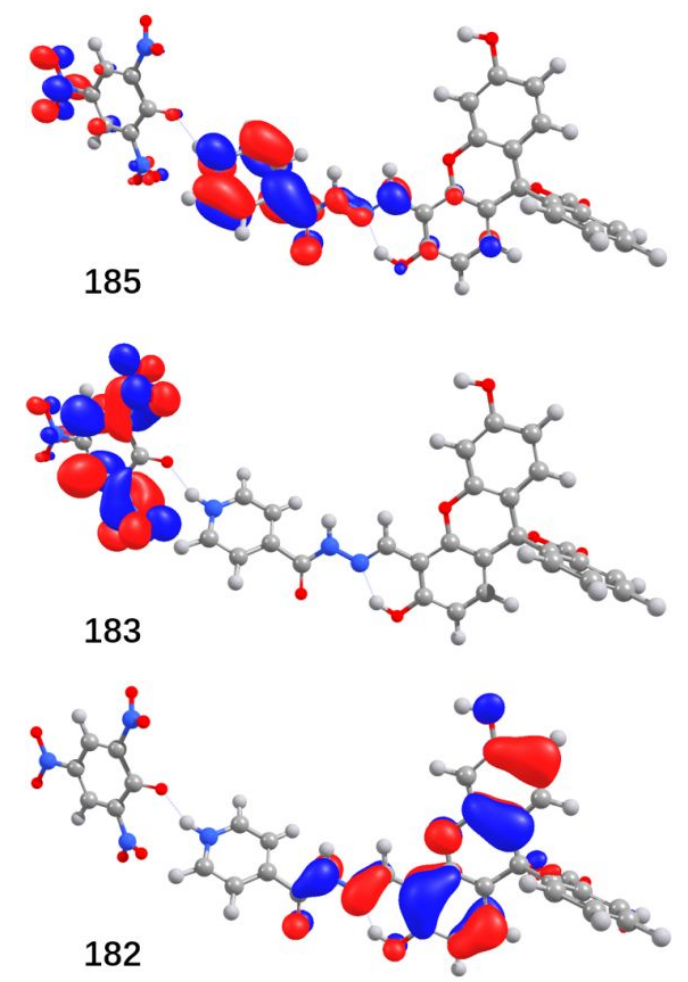

Figure S8. Molecular orbitals related to the excitation process of the $\mathrm{hb}-\mathrm{S}_{0}$ binding model (obtained at CAM-B3LYP/TZVP theory level).
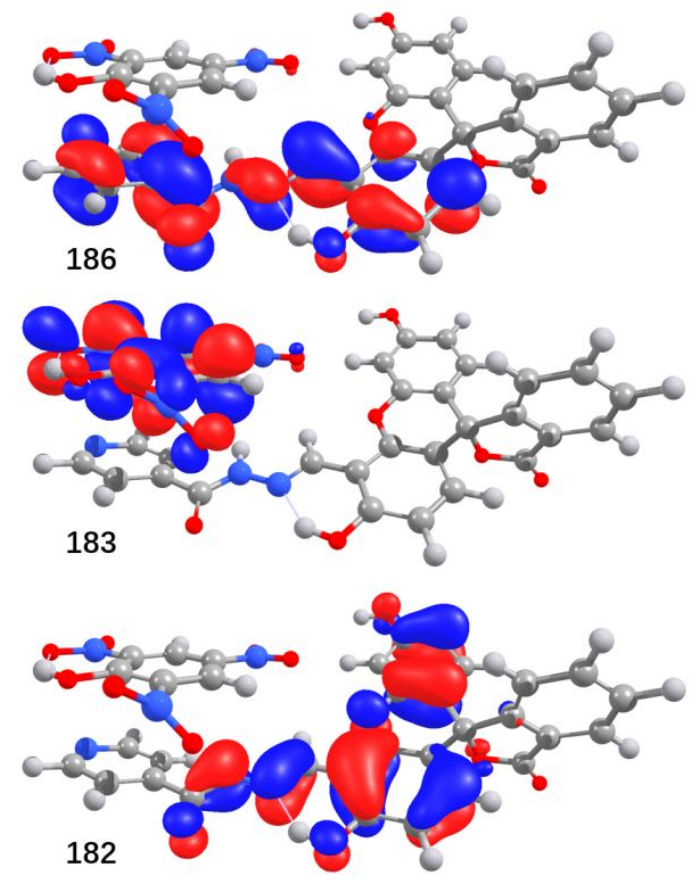

Figure S9. Molecular orbitals related to the excitation process of the $\mathrm{pp}-\mathrm{S}_{0}$ binding model (obtained at CAM-B3LYP/TZVP theory level). 


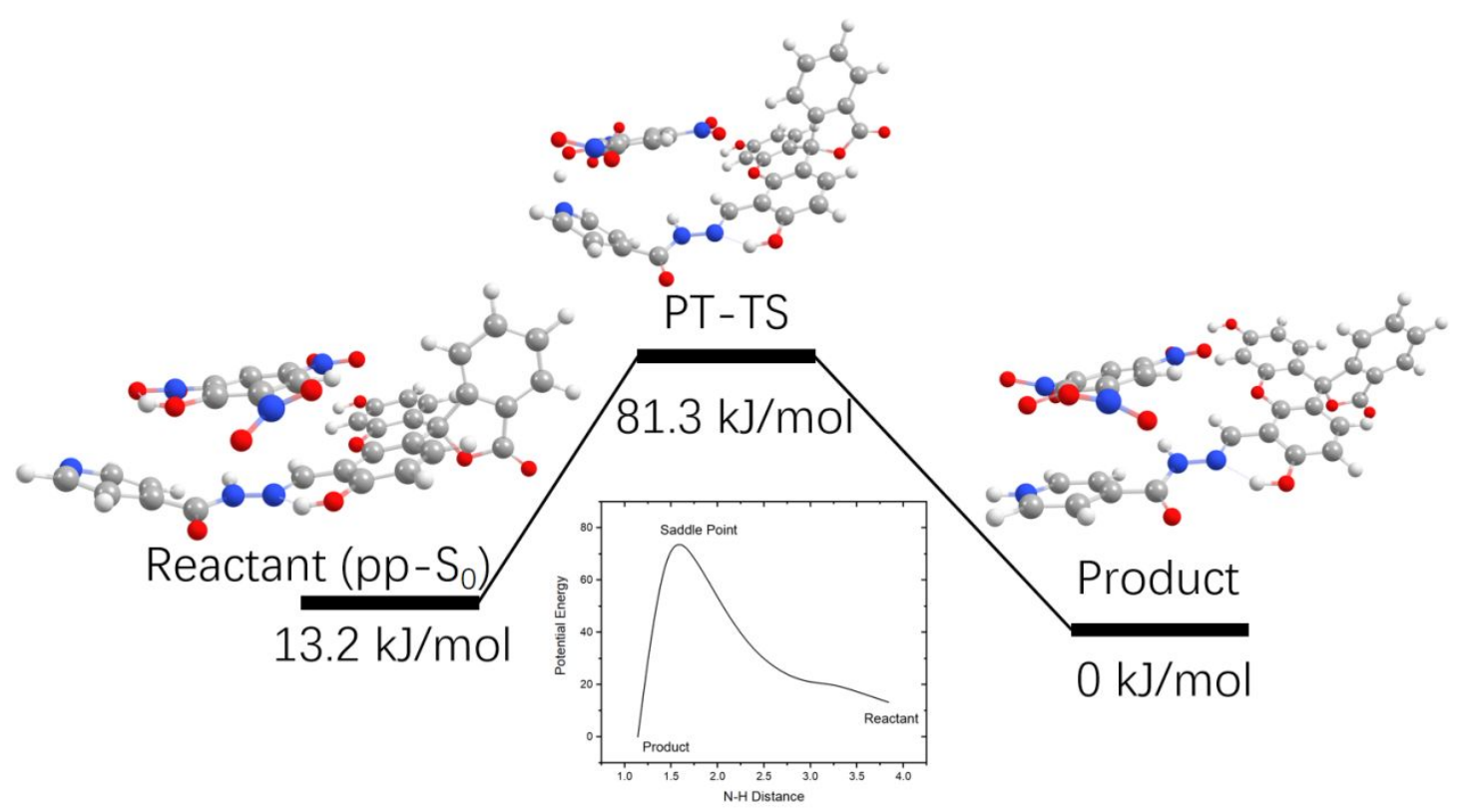

Figure S10. Potential energy curves and energy diagram of the proton transfer process for the $\mathrm{pp}-\mathrm{S}_{0}$ model.

Table S1. Excitation and emission energies of the sensor at CAM-B3LYP/TZVP theory level.

\begin{tabular}{cccc}
\hline & Contribution & Energy & $\mathrm{f}$ \\
\hline $\mathrm{S}_{0} \rightarrow \mathrm{S}_{1}$ & $124(\mathrm{H}) \rightarrow 125(\mathrm{~L})(82 \%)$ & $303 \mathrm{~nm}$ & 0.8784 \\
$\mathrm{~S}_{0} \rightarrow \mathrm{S}_{2}$ & $123 \rightarrow 125(71 \%)$ & $287 \mathrm{~nm}$ & 0.2268 \\
& $\mathbf{S}_{\mathbf{1}}$ & $395 \mathrm{~nm}$ & 1.1482 \\
$\mathrm{~S}_{1} \rightarrow \mathrm{S}_{0}$ & $125(\mathrm{~L}) \rightarrow 124(\mathrm{H})\left(\begin{array}{r}(91 \%) \\
\mathbf{S}_{\mathbf{1}}-\mathbf{P T}\end{array}\right.$ & $452 \mathrm{~nm}$ & 0.2212 \\
\hline $\mathrm{S}_{1} \rightarrow \mathrm{S}_{0}$ & $125(\mathrm{~L}) \rightarrow 124(\mathrm{H})(95 \%)$ & & \\
\hline
\end{tabular}

Table S2. Energy decomposition results for $\mathrm{pp}-\mathrm{S}_{0}$.

\begin{tabular}{cc}
\hline Force & Energy $(\mathrm{kJ} / \mathrm{mol})$ \\
\hline Electrostatics & -12.1 \\
Exchange & 61.8 \\
Induction & -12.1 \\
Dispersion & -77.9 \\
Total & -40.3 \\
\hline
\end{tabular}

